\title{
Explicit Feedback, Implicit Feedback and Learner Beliefs on Corrective Feedback: Does Cultural Factor Influence Learner's Preference of Corrective Feedback?
}

\author{
Yi Wang \\ College of Foreign languages, Nanjing University of Aeronautics and Astronautics, China
}

\begin{abstract}
This study compared the learner preferences for particular types of error correction between two distinct cultural groups in the foreign language learning context. The learners of English from a Chinese university and L2 Chinese Learners from an American university participated in the study. Repeated measures ANOVAs and Pairwise comparison revealed that certain background factors that may predict beliefs and some beliefs about feedback may be shared across the cultures.
\end{abstract}

Keywords: learner's belief, explicit corrective feedback, implicit corrective feedback

\section{Introduction}

Corrective feedback facilitates L2 learning and it helps to direct learners' attention to second language form. It is defined as a teacher's reactive move that invites a learner to attend to the grammatical accuracy of the utterance which is produced by the learner. Corrective feedback in the classroom involves a complicated and dynamic process of human interaction involves linguistic, cognitive, psycholinguistic and pragmatic choices by both the learner and the teacher. The current study investigates the cross-cultural influences on the learners' preferences for particular forms of error correction. By analyzing learner preferences for particular types of error correction between two distinct cultural groups in the EFL context, it tries to find some patterns which may raise teacher awareness. It will also help solve the discrepancy between the teacher's intensions of the feedback and student's understandings of those intensions.

\section{Learner beliefs on corrective feedback}

Investigating learner beliefs regarding corrective feedback is of value theoretically and pedagogically for two reasons. First, learner beliefs are directly related to their learning behaviors, which subsequently influence learning outcomes (Mori, 1999). Therefore, researching learner 
beliefs ultimately leads to more effective teaching methods. Second, mismatch between teachers' intensions and learners interpretation of those intensions may result in negative effects on learning (Kim \& Han, 2007).

The present study examines learner beliefs on corrective feedback and if there is evidence for preferences in specific forms of error correction between cultural groups.

\section{Cultural influence on learner beliefs on corrective feedback}

Learner beliefs towards corrective feedback may be affected by learner's cultural backgrounds. Although learner beliefs about corrective feedback are generally positive, some studies show that the extent to which they have a positive view of corrective feedback varies depending on learner's cultural backgrounds.

Schulz (2001) administered questionnaires to the teachers and students in the Unites States and in Columbia. It is found that grammar instruction was considered more important than by teachers and students in the United States. She attributed these results to (a) the learning background of foreign language learners who were taught with grammar translation method, (b) a mythical belief passed on from past, and (c) their own learning experience in which they benefit from explicit grammar instruction, including corrective feedback.

In this study, American and Chinese cultures are two distinctive cultures. American culture is explicit culture and Chinese culture is implicit. Edward T. Hall (Beyond culture, 1976) finds that some cultures are communicating with a high context and some cultures use a low context for their daily communication. American culture is a low context culture. They say what they think and think what they say, messages are sent explicitly. They expect the same thing from their communication partner, so they avoid any misunderstandings through interpretation. China is a typical example for high context culture. Messages are sent implicitly. There is space for interpretation. Besides, American culture encourages individualism and equality, which is reflected in the student-centered classroom. Chinese culture is high social hierarchical culture which is reflected in the teacher-centered classroom. These cultural differences may influence the classroom interaction, especially the error correction between students and teachers.

\section{Corrective feedback}

Corrective feedback provides positive evidence and negative evidence which are believed to be crucial in second language development. Positive evidence provides learners with correct and target like structures or what is acceptable in L2 while negative evidence warns learners against what is unacceptable. Corrective feedback enables the learner to confirm, disconfirm, and where necessary modify the hypothetical, transitional rules of his interlanguage.

According to Ellis, Loewen and Erlam (2006), corrective feedback takes the form of one or combination of the following responses by a teacher when a learner makes an error: (1)an indication that the learner committed an error, (2) the provision of correct form of the error, and (3) the provision of some metalingual explanation regarding the error.

The most comprehensive taxonomy of corrective feedback has been provided by Lyster and Ranta(1997) who classified corrective feedback into six categories, which are explicit correction, recast, metalinguistic feedback, elicitation, repetition and clarification request. 


\section{Implicit and explicit feedback}

Different types of corrective feedback can be categorized according to their degree of implicitness or explicitness. Implicit types of corrective feedback are those which do not explicitly warn learners regarding their error and thus do not disrupt the flow of communication. Explicit corrective feedback, on the other hand, explicitly demands learners to pay attention to an incorrect feature in their output and thus is likely to stop the communication.

Recast is usually considered as implicit corrective feedback by providing learners mainly with positive evidence, explicit correction and metalinguistic feedback is more explicit by indicating learners the nature of the error and providing them with explicit negative evidence. Elicitation, repetition and clarification request fall into the category of prompts. Prompt use a range of signals, other than the explicit reformulation of the error to push learner to self repair.

\section{Method}

\subsection{Context and Participants}

The study was conducted in two distinctive cultural contexts. The Chinese learners of English in the current study lived in a typical foreign language environment. They were educated in traditional foreign language classroom which is form oriented and teacher centered. The Chinese socioeducational environment consists of a test-driven culture.

Twenty-three L2 Chinese Learners aged 18-24 from an American university participated in the study. Twenty-one were native speakers of English and two reported Korean as their native language. At the time of data collection, they were in their $2^{\text {nd }}, 3^{\text {rd }}$ semesters of their Chinese study. They were also educated in a foreign language classroom which is communication oriented and student centered. The American socioeducational environment encourages creativity and individuality.

\subsection{Procedure}

The participants were asked to complete two questionnaires (one version for Chinese participants, one version for American Participants). Questionnaire 1 was to get their general attitudes about error correction as language learner and some information about their bio and training history. Questionnaire 2 was feedback preference analysis.

The feedback preference analysis questionnaire consisted of six 4-point Liker Scale questions that asked participants to rate different corrective feedback examples according to "how helpful" they felt it was in correcting the modeled error( $1=$ very good, $2=$ good, $3=$ bad, $4=$ very bad $)$. The audio recording first presented in the initial dialogue between student and teacher which consisted an erroneous utterance by the student. Each of the six different possible corrective attempts made by the teacher was then presented. Each example response was followed by a 10 -second pause allowing participants to make their selection.

The six distinct forms of corrective feedback were outlined by Lyster and Ranta (1997).They were explicit correction, recast, metalinguistic feedback, elicitation, repetition, clarification request. For the purposes of data analysis these feedback types would be looked at individually or grouped 
into similar feedback families.

\section{Results}

\subsection{Data conversion and feedback classification}

The data of the Likert scale selections from Corrective feedback Analysis Questionaire were converted to values by attributing points to the selection. 1( very good) was allocated 4 points, 2 (good) 3 points, 3 (bad) 2 points, and 1(very bad) 1 point.

The six types of corrective feedback were grouped into three categories (implicit feedback, explicit feedback, and prompts) based on sharing similar characteristics. The analysis of results would be more clearly interpretable and convenient for the comparison of the three categories. Participants now had three mean scores to analyze rather than 6 different scores.

\subsection{Feedback Preferences within Cultural Groups}

First, whether there are significant differences within each cultural group for each of the three feedback categories (implicit feedback, explicit feedback, and prompts) was analyzed.

Repeated measures ANOVAs were first used to assess if a significant main effect existed for feedback categories within each cultural group. Pairwise comparison was then used to examine comparisons between specific categories to help spot where significant differences occurred (explicit-prompts, explicit-implicit, prompts-implicit).

\subsection{American student feedback preference}

American students rated explicit feedback hightest (3.13), followed by implicit feedback(2.87) and then prompts(2.09). A one-way repeated measured analysis of variance was conducted. The result indicated a significant corrective feedback effect, $F(1,22)=11.56, p<.05$. Follow up comparisons indicated that there were significant difference between Implicit feedback and prompts $(p=.008)$, and significant difference between explicit feedback and prompts $(p=.0005)$. There was no significant difference between implicit feedback and explicit feedback( $p=.757)$.

\subsection{Chinese student feedback preference}

Chinese students rated explicit feedback hightest (3.19), followed by implicit feedback(2.89) and then prompts(2.23). A one-way repeated measured analysis of variance was conducted. The result indicated a significant corrective feedback effect, $F(1,29)=15.88, p<.01$. Follow up comparisons indicated that there were significant difference between all three feedback family comparisons $(\mathrm{p}=.0001)$.

\subsection{Cultural group preferences for corrective feedback category}

Cultural group feedback preference comparisons were achieved by comparing mean preference rating scores for each feedback category from answers to the Corrective Feedback Analysis Questionnaire. 
Chinese students rated all feedback families higher than their American counterparts. This difference was most marked for recast, less so for explicit correction, and almost identical ratings were indicated for prompts. The analysis of variance revealed a significant interaction effect between feedback family preference and culture for a grammar error between Chinese and American students, $\mathrm{F}(2,50)=6.33, \mathrm{p}<.05$. However, the post hoc comparison confirmed significant feedback preference differences between groups in relation to recasts only $(p=.001)$, although there was near significance for explicit correction $(p=.0705)$

\section{Discussion and conclusion}

Within group analysis indicated that students from both cultural groups ranked the three feedback families in identical fashion, though Chinese Students provided higher overall rankings for all three feedback families. The post-hoc analysis of between-group difference revealed that Chinese students' preference for explicit correction was significantly stronger than that of their American counterparts. American students' preferred recasts significantly less than their Chinese counterparts. The reason may be due to the different instructional focus and learning context each group was accustomed to learning in. Recasts may have appeared more explicit to Chinese students who were accustomed to learning English in form-focused EFL classroom, whereas recasts may have appeared more implicit to American learners in their communicative ESL classes.

As indicated by this study, students from both groups clearly indicated a preference for explicit correction may signal to teachers that this method of correction is one that can be universally appreciated by students across cultures. The use of prompts, although effective in furthering student production of the target language, may not always be the best method, especially in East Asian context where student affective reaction appear to be more of a concern than in Western-based teaching contexts. Contradictory findings, in which Chinese learners indicated a preference to self-correction during interview sessions, yet ranked prompts as their least preferred method of correction on questionnaires, implies that perhaps the direct nature of prompts may cause American Students to feel embarrassed and stressed with having to correct themselves immediately and in front of their peers, despite the fact that they would welcome the opportunity to self-correct. Teachers may want to consider exploring ways which allow learners to self-correct that do not require an immediate reformulation on the part of the student.

The implication for the findings of this study focus essentially on the consideration of cultural and learning contexts when choosing appropriate methods of correction, as well as student relation to correction, which may have been influenced by cultural background. It is imperative that teachers assess what their students' language learning needs are.

\section{Acknowledgements}

The paper is supported by the Fundamental Research Funds for the Central Universities, NO. NR2014032.

\section{References}

[1] Hall, Edward T. Beyond Culture. Garden City, N.Y: Anchor Press, 1976. 
[2] Ellis, R., S. Loewen \& R. Erlam (2006). Implicit and explicit corrective feedback and the acquisition of L2 grammar. Studies in Second Language Acquisition 28.3, 339-368.

[3] Kim, J., \& Han, Z. (2007). Recasts in communicative EFL classes: Do teacher intent and learner interpretation overlap? In A. Mackey (Ed.).Conversation interaction in second language acquisition: A collection of empirical studies (pp. 269-297). Oxford, England: Oxford University Press.

[4] Lyster, R., \& Ranta, L. (1997). Corrective feedback and learner uptake. Studies in Second Language Acquisition, 20, 37-66.

[5] Mori, Y. (1999). Epistemological beliefs and language learning beliefs: What do language learners believe about their learning? Language Learning, 49, 377-415.

[6] Schulz, R. A. (2001). Cultural differences in student and teacher perceptions concerning the role of grammar instruction and corrective feedback: USA - Columbia. The Modern Language Journal, 85(2), 244-258. 